de l'université dans l'évolution et le redéploiement socio-économiques. Après la recherche et l'enseignement, cette troisième mission de l'universisté est celle de la citoyenneté dans le sens le plus large. Elle consacre l'importance de l'apport des sciences à la société, dans le sens de sa contri- bution à un progrès qui ne se conçoit plus seulement dans sa dimension matérielle mais aussi éthique.

$\mathrm{Au}$ moment d'entrer dans le troisième millénaire, il s'agit là d'un des défis les plus importants auxquels l'université et les scientifiques doivent faire face. A cet égard, l'Univer- sité de Liège ne veut être rien moins qu'un exemple. Les actions entreprises par les facultés des Sciences et de Médecine pour développer un pôle d'excellence et de compétences dans le domaine des biotechnologies en sont un des témoignages les plus significatifs

\title{
SCIENCES ET MÉDECINE À NAMUR
}

\section{Maurice Gilbert}

\section{ADRESSE}

M. Gilbert : recteur de l'Université de Namur. Faculté de médecine, 61 , rue de Bruxelles, B5000, Namur, Belgique.

\section{TIRÉS À PART}

\section{Gilbert.}

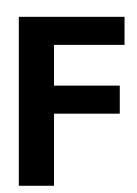

ondées en 1831 par la Compagnie de Jésus, les Facultés Universitaires Notre-Damede-la-Paix sont fréquentées aujourd'hui par quelque 4200 étudiants, répartis entre les six facultés que compte actuellement l'Institution (Philosophie et Lettres, Droit, Sciences économiques, sociales et de gestion, Institut d'Informatique, Sciences, Médecine). Héritières des valeurs issues de la tradition humaniste et jésuite et fortes du pluralisme d'opinion de ses membres, partenaires des acteurs engagés dans l'action sociale, les mutations économiques et la réflexion critique, les Facultés visent l'excellence en donnant priorité à l'homme par attention à la personne, par une approche pluri- et interdisciplinaire des questions à traiter, par l'ouverture à l'universalité. Harmonieusement intégrées à une ville sise sur Meuse et Sambre, au cœur de la Wallonie et de l'Europe en construction, de taille humaine, elles s'emploient, avec les jeunes qu'elles forment, à participer par l'enseignement, la recherche et le service à la communauté, à faire évoluer le monde vers plus d'intelligence, de démocratie et d'humanité, en relevant les défis du monde à venir.

Dans le domaine biomédical, l'enseignement de la candidature en Sciences médicales, d'abord limité à la $1^{\text {re }}$ année, a été complété à partir de 1962 par l'ouverture des $2^{\mathrm{e}}$ et $3^{\mathrm{e}}$ années. La recherche a commencé à ce moment, tant en médecine qu'en biologie. Le département de biologie de la Faculté des Sciences, dont une première grande figure fut le P.A. Bauchau, obtint en 1971 l'autorisation de délivrer les diplômes de deuxième et troisième cycles (licence et doctorat). La recherche s'y développa en conséquence, en collaboration avec la médecine et le département des sciences vétérinaires. Celui-ci organisa l'enseignement des trois candidatures en médecine vétérinaire à la même époque et participe depuis lors à l'effort commun 